\title{
Survey and documentation of anthelmintic plants used in traditional medicine system of tribal communities of Udalguri district of Assam, India
}

\author{
Ananta Swargiary*, Manita Daimari, Mritunjoy Kumar Roy \\ Department of Zoology, Bodoland University, Kokrajhar 783370, India.
}

\begin{tabular}{l}
\hline ARTICLE INFO \\
\hline Received on: 07/08/2019 \\
Accepted on: 10/11/2019 \\
Available online: 03/01/2020
\end{tabular}

Key words:

Anthelmintic, ethnomedicine, traditional healer, Udalguri, Assam.

\begin{abstract}
The present study was aimed to survey and document the anthelmintic medicinal plants traditionally used by the tribal communities of Udalguri district of Assam. The survey was conducted in different villages of the district following a face-to-face interview and a readymade questionnaire. The study found that a total of 75 plant species belonging to 67 genera and 44 plant families were used as deworming agents. The result showed that Andrographis paniculata, Ananas comosus, Hydrocotyle sibthorpioides, and Centella asiatica were the most popular plant species. Acanthaceae family was found to be most common among the traditional healers. The leaves were found to be commonly used plant parts for herbal preparation. Decoction, infusion, and raw preparations were found to be the most commonly used traditional formulation methodologies. The present study could be used to identify the potential anthelmintic plants and in designing new anthelmintic drug having better property and efficacy.
\end{abstract}

\section{INTRODUCTION}

Nature has always been an exemplary source of drugs since ancient times. Medicinal plants continued to be an important therapeutic aid for alleviating ailments of human kind. Ethnobotanical studies are often significant in revealing locally important plant species, especially for the discovery of crude drugs (Muthee et al., 2015). Ethnomedicinal survey of medicinal plants used by traditional medicinal practitioners can form a rich source of data for knowledge about medicinal plants and the ailments for which they are used. Scientists have often found that the herbs themselves, which possess unique combinations of chemical components, are more effective than the chemical derivatives (Shikov et al., 2014). Many developing countries like India rely on plants-based products for treating various diseases, including helminth infection. Medicinal plants are a viable source of parasiticides (Wangchuk et al., 2016). India is a country based on agriculture, and livestock play a significant role for the farmers.

\section{"Corresponding Author}

Ananta Swargiary, Department of Zoology, Bodoland University, Kokrajhar 783370, India.E-mail: ananbuzoo101@gmail.com
The infection with helminthes is still a big problem mainly due to warm temperatures, in association with poor management practices and inadequate control measures (Akhtar et al., 2000). The main goal of present study was the documentation of anthelmintic plants used in traditional medicine system of tribal communities of Udalguri district of Assam, India.

\section{METHODS AND MATERIALS}

\section{Study area and its description}

The present study was carried out in different villages under Udalguri district of Assam. Geographically, it covers an area of $1,852.16 \mathrm{sq} . \mathrm{km}$. According to the 2011 Census report of India, Udalguri district has 802 villages (791 human inhabited and 11 uninhabited) and population size of 832,769 , more than $95 \%$ living in the rural areas.

\section{Data collection and identification of plant samples}

The survey was done during the months of May to November, 2018 and information regarding the anthelmintic plants traditionally used by tribal communities of Udalguri district was collected. The information was collected from different community development block (CDB) with the help 
of informants (village traditional healer or Kaviraja in local language and elderly village people) having ethnomedicinal knowledge. Within every $\mathrm{CDB}$, approximately 20 adjacent villages were taken as a single cluster and one sample (informant) was collected from a cluster. The information was collected in a face-to-face interview manner with the help of readymade questionnaires. Based on the number of villages per CDB, sample size varied from one $\mathrm{CDB}$ to another. Out of $11 \mathrm{CDBs}$, we did not collect any data from two CDBs, namely, Pachim-Mangaldai and Dalgaon-Sialmari because of less numbers of village(s). The information collected from informants mainly includedinformer's bio-data, plant(s) part(s) used, local name of plant(s), traditional formulations, and mode of administration. The plants were collected as per the information given by the informer. A total of 42 informants were interviewed from 39 different villages. One informant was interviewed from each of the 36 villages, while two informants were collected from Pakribari, Barnagaon, and No. 2 Kadabil. The various information collection sites under different $\mathrm{CDB}$ were represented in Figure 1. Based on the information provided by the informants, sample plants were collected and processed for identification. Herbarium sheet were prepared and submitted to the Department of Botany, Bodoland University for identification.

\section{Data analysis}

Following quantitative analysis was carried out to assess the importance of medicinal plants following Hussain et al. (2018).
Frequency of Citation (FC): It is the number of informants who mentioned a certain species.

Relative frequency of citation (RFC): It is obtained by dividing FC by total number of informants $(N)$. The value of RFC indicates the citing percentage of each species of medicinal plants. RFC was calculated by using the following formula (Tardio et al., 2008):

$$
\mathrm{RFC}=\mathrm{FC} / N
$$

The value of RFC varies from zero (when nobody cites to a plant as important) to one (when all the informants consider a certain species important).

Family importance value (FIV): FIV indicates the local importance of the families of plant species and is calculated by counting the percentage of informants mentioning a specific family (Vitalini et al., 2013).

$$
\mathrm{FIV}=[\mathrm{FC} \text { (family) } / N] \times 100
$$

\section{Statistical analysis}

All the statistical calculations, graphs, etc. were carried out in Microsoft excel and Origin software.

\section{RESULTS AND DISCUSSIONS}

\section{Demography of informants and collection sites}

Traditional knowledge system of herbal medicines based on the plant product is a healthy practice among the tribal

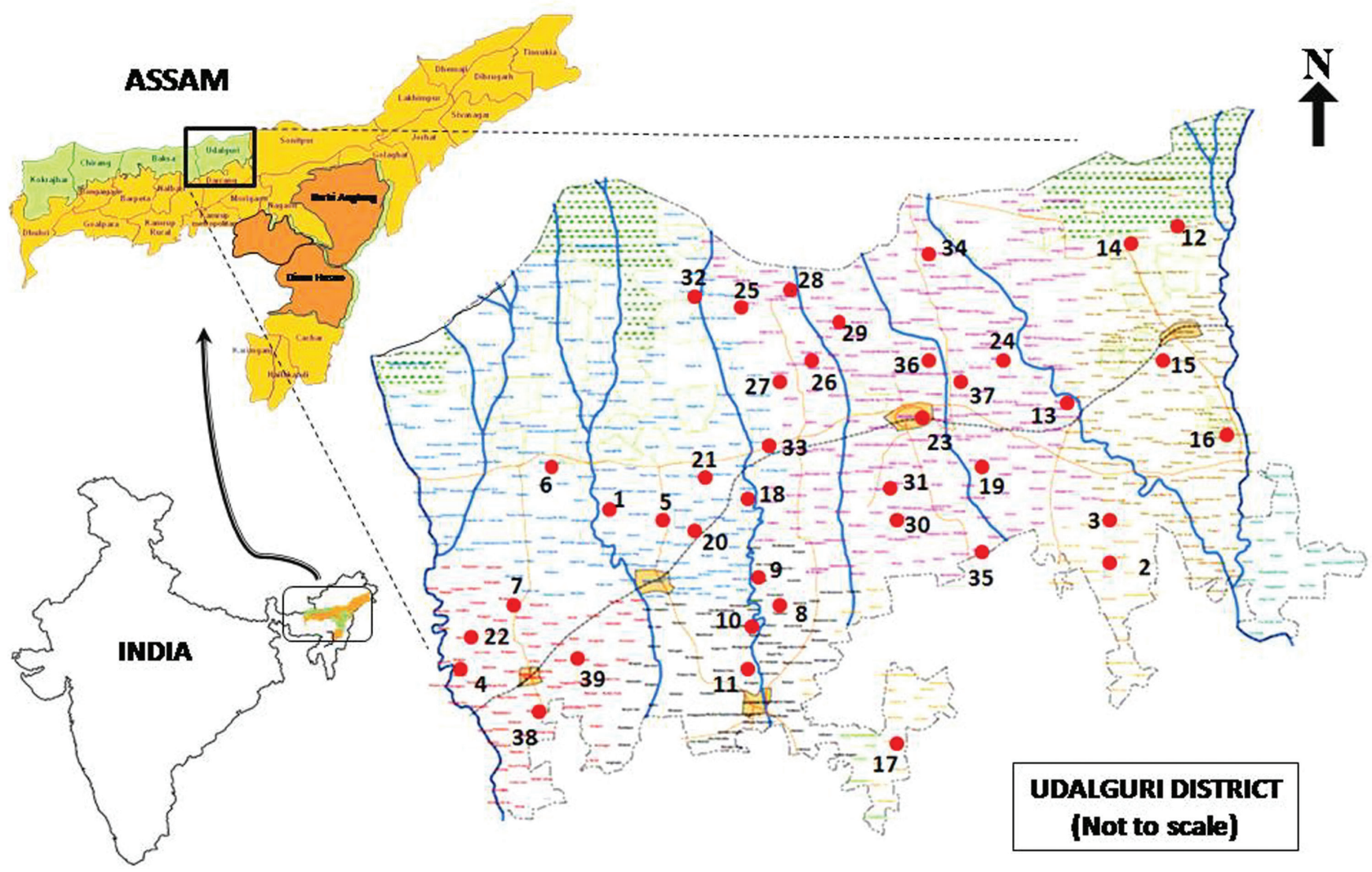

Figure 1. Map of Udalguri district of Assam and sample collection sites. Red dots represent the villages where data has been collected. [Numbers represents the serial no. of villages of Table 1]. 
communities of India. Living in the rural areas and far away from towns and cities, economically downtrodden tribal communities depends on plant-based medicines for common diseases. In our present study, we selected 39 sample villages from 9 CDBs and 42 numbers of traditional informants have been interviewed. The names of CDBs, villages, its geographical location, and demography of the selected informants were presented in Tables 1 and 2, respectively. Out of 9 CDBs, a highest of 12 villages was visited from Udalguri $\mathrm{CDB}$ and 14 numbers of informants were interviewed. However, only one informant was collected each from Borchola and Pub-Mangaldai CDBs.

A total of 39 villages were surveyed with the help of local people via the administration of semi-structured interviews and ready-made questionnaire (Table 1). A total of 42 informants were interviewed out of which $64 \%$ constitute the male informants (Table 2). Among the informants, $83 \%$ is traditional healer who possess the ethnomedicinal knowledge and practice regularly and earn little amount of money. Seventeen percent of the informants are the elderly people who do not practice but possess ethnomedicinal knowledge gathered from their forefathers. It is also seen that most of the traditional knowledge bearers are above 40-year old. Very few (about 12\%) were found to be below 40 years of age. In terms of literacy rate, about $79 \%$ informants were having formal education, while $21 \%$ were illiterates. Among the literates, $19 \%$ were having college level education, while $60 \%$ have school level education. However, compared to females, the males were found to be more educated. Out of 27 numbers of male informants, $85 \%$ are found to be educated. Meanwhile, the female literacy was found to be about $60 \%$ of the 15 female informants. Among 42 informants, very few $(18 \%)$ have government salaried job. In terms of plant citations, however, the literates and illiterates cited more or less similar number of plants, an average of 4.4 citations/informants and 4.2 citations/informants, respectively. The names of the plant species, its local name, habit, habitat, parts used, and traditional formulation of the herbal medicine is shown in the Table 3.

Use of medicinal plants for the treatment of diseases is a common practice to the rural, economically, and educationally backward tribal communities of India (Fayaz et al., 2019; Singh et al., 2017). Most of the times traditional medicine (TM) Systems do not have any form of written documents and are transferred orally from generation to generation. Like many other parts of the worlds, in Indian subcontinent also, TMs are practiced mainly by uneducated, poor, old, and aged people. It is also reported in many survey reports that male folks act as the major information bearer than females. Our study also revealed similar kind of traditional knowledge bearers where most of the informants are aged and male individualsx (Ritter et al., 2012; Teklehaymanot, 2017). However, unlike many other countries, such as Kenya, and Pakistan, where TMs are reported to be practiced mostly by illiterates, we found dissimilar result with most of the informants (79\%) having formal education (Ahmad et al., 2015; Maphosa and Masika, 2010). Fayaz et al. (2019) also found that $71 \%$ of the informants from Jakholi Block of district Rudraprayag, Uttarakhand state were having formal education. It has also been seen that out of 42 informants $18 \%$ were having government salaried jobs, and none of them practice professional herbal treatment and they gathered their ethnomedicinal knowledge from their forefathers. Most of the traditional healers make some business out of their ethnomedicinal
Table 1. Name of the CDB, villages and geographical locations of survey sites.

\begin{tabular}{|c|c|c|c|}
\hline SI. no. & Name of the CDB & Villages & Geographical location \\
\hline 1. & Borchola & 1. Dhupguri & $26^{\circ} 39^{\prime} 49^{\prime \prime} \mathrm{N} 91^{\circ} 45^{\prime} 00^{\prime \prime} \mathrm{E}$ \\
\hline \multirow[t]{2}{*}{2.} & Bechimari & 2. Koraibari & $26^{\circ} 39^{\prime} 31^{\prime \prime} \mathrm{N} 92^{\circ} 15^{\prime} 10^{\prime \prime} \mathrm{E}^{*}$ \\
\hline & & 3. Aitharjhar & $26^{\circ} 41^{\prime} 05^{\prime \prime} \mathrm{N} 92^{\circ} 15^{\prime} 11^{\prime \prime} \mathrm{E}^{*}$ \\
\hline \multirow[t]{4}{*}{3.} & Bhergaon & 4. Barigaon & $26^{\circ} 35^{\prime} 35^{\prime \prime} \mathrm{N} 91^{\circ} 46^{\prime} 12^{\prime \prime} \mathrm{E}$ \\
\hline & & 5. Ratanpur & $26^{\circ} 42^{\prime} 29^{\prime \prime} \mathrm{N} 91^{\circ} 55^{\prime} 06^{\prime \prime} \mathrm{E}$ \\
\hline & & 6. Dimakuchi & $26^{\circ} 44^{\prime} 43^{\prime \prime} \mathrm{N} 91^{\circ} 50^{\prime} 22^{\prime \prime} \mathrm{E}$ \\
\hline & & 7. Bhergaon & $26^{\circ} 41^{\prime} 19^{\prime \prime} \mathrm{N} 91^{\circ} 51^{\prime} 52^{\prime \prime} \mathrm{E}$ \\
\hline \multirow[t]{4}{*}{4.} & Kalaigaon & 8. Dumaruguri & $26^{\circ} 39^{\prime} 56^{\prime \prime} \mathrm{N} 91^{\circ} 59^{\prime} 54^{\prime \prime} \mathrm{E}$ \\
\hline & & 9. Kuiyabil & $26^{\circ} 40^{\prime} 48^{\prime \prime} \mathrm{N} 91^{\circ} 59^{\prime} 12^{\prime \prime} \mathrm{E}$ \\
\hline & & 10. Balipara & $26^{\circ} 38^{\prime} 05^{\prime \prime} \mathrm{N} 91^{\circ} 58^{\prime} 39^{\prime \prime} \mathrm{E}$ \\
\hline & & 11. Sagunbahi & $26^{\circ} 36^{\prime} 30^{\prime \prime} \mathrm{N} 91^{\circ} 58^{\prime} 55^{\prime \prime} \mathrm{E}$ \\
\hline \multirow[t]{5}{*}{5.} & Mazbat & 12. Adarsho & $26^{\circ} 15^{\prime} 33^{\prime \prime} \mathrm{N} 92^{\circ} 16^{\prime} 22^{\prime \prime} \mathrm{E}$ \\
\hline & & 13. Dhansri Gat & $26^{\circ} 38^{\prime} 07^{\prime \prime} \mathrm{N} 92^{\circ} 18^{\prime} 09^{\prime \prime} \mathrm{E}$ \\
\hline & & 14. No. 2 Kadabil & $26^{\circ} 51^{\prime} 27^{\prime \prime} \mathrm{N} 92^{\circ} 18^{\prime} 34^{\prime \prime} \mathrm{E}^{* *}$ \\
\hline & & 15. Gerubari & $26^{\circ} 46^{\prime} 18^{\prime \prime} \mathrm{N} 92^{\circ} 17^{\prime} 56^{\prime \prime} \mathrm{E}$ \\
\hline & & 16. Orang & $26^{\circ} 42^{\prime} 25^{\prime \prime} \mathrm{N} 92^{\circ} 19^{\prime} 37^{\prime \prime} \mathrm{E}$ \\
\hline 6. & Pub-Mangaldai & 17. Kacharipara & $26^{\circ} 39^{\prime} 27^{\prime \prime} \mathrm{N} 91^{\circ} 46^{\prime} 44^{\prime \prime} \mathrm{E}$ \\
\hline \multirow[t]{5}{*}{7.} & Rowta & 18. Kathalguri & $26^{\circ} 42^{\prime} 57^{\prime \prime} \mathrm{N} 91^{\circ} 58^{\prime} 46^{\prime \prime} \mathrm{E}$ \\
\hline & & 19. Goraibari & $26^{\circ} 43^{\prime} 22^{\prime \prime} \mathrm{N} 92^{\circ} 07^{\prime} 48^{\prime \prime} \mathrm{E}$ \\
\hline & & 20. Panipota & $26^{\circ} 42^{\prime} 04^{\prime \prime} \mathrm{N} 91^{\circ} 55^{\prime} 54^{\prime \prime} \mathrm{E}$ \\
\hline & & 21. No.1 Bhergaon & $26^{\circ} 43^{\prime} 41^{\prime \prime} \mathrm{N} 92^{\circ} 09^{\prime} 40^{\prime \prime} \mathrm{E}$ \\
\hline & & 22. Gopsachuba & $26^{\circ} 38^{\prime} 06^{\prime \prime} \mathrm{N} 91^{\circ} 46^{\prime} 02^{\prime \prime} \mathrm{E}$ \\
\hline \multirow[t]{15}{*}{8.} & Udalguri & 23. Deolguri & $26^{\circ} 44^{\prime} 13^{\prime \prime} \mathrm{N} 92^{\circ} 00^{\prime} 21^{\prime \prime} \mathrm{E}$ \\
\hline & & 24. Ahomakha & $26^{\circ} 47^{\prime} 30^{\prime \prime} \mathrm{N} 92^{\circ} 10^{\prime} 36^{\prime \prime} \mathrm{E}$ \\
\hline & & 25. Kundarbil (No. 3), Amjuli & $26^{\circ} 46^{\prime} 53^{\prime \prime} \mathrm{N} 92^{\circ} 01^{\prime} 21^{\prime \prime} \mathrm{E}$ \\
\hline & & 26. Uttar Nalbari (Borigaon) & $26^{\circ} 47^{\prime} 46^{\prime \prime} \mathrm{N} 92^{\circ} 02^{\prime} 12^{\prime \prime} \mathrm{E}$ \\
\hline & & 27. Panimudijhar & $26^{\circ} 47^{\prime} 47^{\prime \prime} \mathrm{N} 92^{\circ} 00^{\prime} 34^{\prime \prime} \mathrm{E}$ \\
\hline & & 28. Kundarbil-No. 1 & $26^{\circ} 50^{\prime} 22^{\prime \prime} \mathrm{N} 91^{\circ} 59^{\prime} 12^{\prime \prime} \mathrm{E}$ \\
\hline & & 29. Pakribari & $26^{\circ} 46^{\prime} 51^{\prime \prime} \mathrm{N} 92^{\circ} 02^{\prime} 57^{\prime \prime} \mathrm{E}^{* *}$ \\
\hline & & 30. Khatorbari & $26^{\circ} 42^{\prime} 14^{\prime \prime} \mathrm{N} 92^{\circ} 04^{\prime} 46^{\prime \prime} \mathrm{E}$ \\
\hline & & 31. Barnagaon & $26^{\circ} 42^{\prime} 22^{\prime \prime} \mathrm{N} 92^{\circ} 54^{\prime} 00^{\prime \prime} \mathrm{E}^{* *}$ \\
\hline & & 32. Majuli Basti & $26^{\circ} 51^{\prime} 44^{\prime \prime} \mathrm{N} 91^{\circ} 55^{\prime} 28^{\prime \prime} \mathrm{E}$ \\
\hline & & 33. Sonai bathabari & $26^{\circ} 48^{\prime} 01^{\prime \prime} \mathrm{N} 92^{\circ} 06^{\prime} 43^{\prime \prime} \mathrm{E}$ \\
\hline & & 34. Jamuguri & $26^{\circ} 43^{\prime} 17^{\prime \prime} \mathrm{N} 92^{\circ} 16^{\prime} 54^{\prime \prime} \mathrm{E}$ \\
\hline & & 35. Medhipara & $26^{\circ} 40^{\prime} 29^{\prime \prime} \mathrm{N} 92^{\circ} 10^{\prime} 08^{\prime \prime} \mathrm{E}$ \\
\hline & & 36. Sonai Alisinga & $26^{\circ} 79^{\prime} 86^{\prime \prime} \mathrm{N} 92^{\circ} 12^{\prime} 16^{\prime \prime} \mathrm{E}^{*}$ \\
\hline & & 37. Niz sonai & $26^{\circ} 79^{\prime} 40^{\prime \prime} \mathrm{N} 92^{\circ} 12^{\prime} 40^{\prime \prime} \mathrm{E}^{*}$ \\
\hline \multirow[t]{2}{*}{9.} & Khoirabari & 38. Batabari & $26^{\circ} 35^{\prime} 25^{\prime \prime} \mathrm{N} 91^{\circ} 49^{\prime} 01^{\prime \prime} \mathrm{E}$ \\
\hline & & 39. Moholiapara & $26^{\circ} 37^{\prime} 29^{\prime \prime} \mathrm{N} 91^{\circ} 51^{\prime} 45^{\prime \prime} \mathrm{E}$ \\
\hline
\end{tabular}

Serial number indicates the information collection sites as shown in the map.

$\mathrm{CDB}=$ community development block.

*No report of anthelmintic plant use, **Villages from where two informants were collected.

knowledge by making small packets and mixtures, which they sell to other villagers. During our survey, we have also come across many people who visited the traditional healer in their homes to purchase the herbal preparation.

\section{Anthelmintic medicinal plants used by tribal communities}

A total of 160 plant citations were recorded from 42 informants (Table 3). Out of total plant citations, 32 plants were found to be reported more than once by the local traditional healers. 
Table 2. Demography of informants from different villages of Udalguri district of Assam.

\begin{tabular}{|c|c|c|c|c|c|c|c|c|c|}
\hline \multirow{2}{*}{ Name of CDB } & \multirow{2}{*}{ Traditionalhealer } & \multirow{2}{*}{ Elderly person } & \multirow{2}{*}{ Male } & \multirow{2}{*}{ Female } & \multicolumn{2}{|c|}{ Age (in years) } & \multicolumn{3}{|c|}{ Literacy } \\
\hline & & & & & $40-50$ & $>\mathbf{5 0}$ & School level & College level & Illiterate \\
\hline Udalguri & 14 & 3 & 12 & 5 & 5 & 10 & 10 & 4 & 3 \\
\hline Khairabari & 2 & - & 1 & 1 & - & 1 & 1 & 1 & - \\
\hline Rowta & 5 & - & 4 & 1 & 4 & 1 & 4 & - & 1 \\
\hline Pub- Mangaldoi & 1 & - & - & 1 & 1 & - & 1 & - & - \\
\hline Mazbat & 5 & 1 & 4 & 2 & 1 & 5 & - & 3 & 3 \\
\hline Bechimari & - & 2 & 1 & 1 & - & - & 2 & - & - \\
\hline Bhergaon & 4 & - & 3 & 1 & - & 4 & 3 & - & 1 \\
\hline Kalaigaon & 4 & - & 1 & 3 & 2 & 2 & 3 & - & 1 \\
\hline Borchala & - & 1 & 1 & - & 1 & - & 1 & - & - \\
\hline Total & 35 & 7 & 27 & 15 & 14 & 23 & 25 & 8 & 9 \\
\hline
\end{tabular}

$\mathrm{CDB}=$ community development blocks.

Table 3. List of medicinal plants used in the traditional medicine system against helmintic infection by tribal communities of Udalguri district of Assam with their identification numbers.

\begin{tabular}{|c|c|c|c|c|c|c|c|c|c|}
\hline Sl. No. & Scientific Name & Family & Local name & Habit & Habitat & Parts used & Formulation & FC & RFC \\
\hline 1. & $\begin{array}{l}\text { Persicaria strigosa (R. Br.) Nakai } \\
\text { [BUBH000021] }\end{array}$ & Polygonaceae & Alari gwja & Herb climber & $\mathrm{Wd}$ & $\mathrm{L}$ & Raw & 1 & 0.0238 \\
\hline 2. & Aloe vera (L.) Burm.f. [BUBH0000022] & Xanthorrhoeaceae & Aloevera & Herb & Do & $\mathrm{L}, \mathrm{J}$ & Raw & 1 & 0.0238 \\
\hline 3. & Phyllanthus emblica L. [BUBH0000023] & Euphorbiaceae & Amla & Tree & Do & $\mathrm{F}$ & Raw & 2 & 0.047 \\
\hline 4. & $\begin{array}{l}\text { Tinospora cordifolia (Willd.) Miers } \\
\text { [BUBH0000024] }\end{array}$ & Menispermaceae & Amor lata & Climber & $\mathrm{Wd}$ & St & Raw & 4 & 0.095 \\
\hline 5. & Ananas comosus (L.) Merr. [BUBH0000025] & Bromeliaceae & Anaras & Herb & Do & $\mathrm{L}$ & Raw & 9 & 0.214 \\
\hline 6. & Morinda angustifolia Roxb. [BUBH0000123] & Rubiaceae & Asho & Small tree & Do & $\mathrm{R}$ & Decoction & 1 & 0.0238 \\
\hline 7. & Litsea glutinosa Lour [BUBH0000087] & Lauraceae & Baghnala & Tree & $\mathrm{Wd}$ & $\mathrm{B}$ & Decoction & 2 & 0.047 \\
\hline 8. & $\begin{array}{l}\text { Terminalia bellirica (Gaertn.) Roxb. } \\
\text { [BUBH0000069] }\end{array}$ & Combretaceae & Bhaora & Tree & Wd & $\mathrm{F}$ & Raw & 3 & 0.071 \\
\hline 9. & Aegle marmelos (L.) Correa [BUBH0000068] & Rutaceae & Bell & Tree & Do & $\mathrm{L}$ & Decoction & 3 & 0.071 \\
\hline 10. & Prunus persica (L.) Stokes [BUBH0000088] & Rosaseae & Bon bwigri, Thaijou khohe & Tree & Do & $\mathrm{L}$ & Raw & 1 & 0.0238 \\
\hline 11. & Scoparia dulcis L. [BUBH0000089] & Plantagenaceae & $\begin{array}{l}\text { Bongfang rakheb, Sini } \\
\text { dongfang }\end{array}$ & Herb & Wd & $\mathrm{L}$ & Decoction & 1 & 0.0238 \\
\hline 12. & $\begin{array}{l}\text { Leucas aspera (Willd.) Link. } \\
\text { [BUBH0000010] }\end{array}$ & Lamiaceae & Brahmaputra, Kansinsa & Herb & $\mathrm{Wd}$ & $\mathrm{L}$ & Raw & 1 & 0.0238 \\
\hline 13. & $\begin{array}{l}\text { Zingiber zerumbet (L.) Roscoe ex Sm. } \\
\text { [BUBH0000090] }\end{array}$ & Zingiberaceae & Bura uth & Herb & $\mathrm{Wd}$ & $\mathrm{T}$ & Infusion & 1 & 0.0238 \\
\hline 14. & Ziziphus mauritiana Lam. [BUBH0000030] & Rhamnaceae & Bwigri & Shrub & Do & $\mathrm{L}$ & Raw & 3 & 0.071 \\
\hline 15. & $\begin{array}{l}\text { Lippia alba (Mill.) N.E.Br. ex Britton \& } \\
\text { P.Wilson [BUBH0000056] }\end{array}$ & Verbenaceae & Bwrma dari, Onthai bajab & Shrub & $\mathrm{Wd}$ & $\mathrm{L}$ & Decoction & 1 & 0.0238 \\
\hline 16. & Cocos nucifera L. [BUBH0000091] & Arecaceae & Narengkol & Tree & Do & En & Decoction & 1 & 0.0238 \\
\hline 17. & Punica granatum L. [BUBH0000031] & Lythraceae & Dalim & Shrub & Do & $\mathrm{B}(\mathrm{R})$ & Raw & 2 & 0.047 \\
\hline 18. & $\begin{array}{l}\text { Cinnamomum verum J.S. Presl } \\
\text { [BUBH0000092] }\end{array}$ & Lauraceae & Dalsini & Tree & Do & $\mathrm{B}$ & Raw & 1 & 0.0238 \\
\hline 19. & $\begin{array}{l}\text { Clerodendrum indicum (L.) Kuntze } \\
\text { [BUBH0000093] }\end{array}$ & Lamiaceae & Ekhlabwr & Shrub & $\mathrm{Wd}$ & $\mathrm{L}, \mathrm{Fl}$ & Raw & 3 & 0.071 \\
\hline 20. & Premna herbacea Roxb. [BUBH0000094] & Verbenaceae & Gadeb (keradapini) & Herb & Wd & $\mathrm{L}$ & Decoction & 1 & 0.0238 \\
\hline 21. & $\begin{array}{l}\text { Calotropis gigantea }(\mathrm{L} .) \text { Dryand. } \\
{[\mathrm{BUBH} 0000072]}\end{array}$ & Apocynaceae & Gogondo, Ogango & Shrub & $\mathrm{Wd}$ & $\mathrm{L}$ & Raw & 1 & 0.0238 \\
\hline 22. & Piper nigrum L. [BUBH0000035] & Piperaceae & Golmoris & Shrub & Do & $\mathrm{S}$ & Raw & 2 & 0.047 \\
\hline 23. & $\begin{array}{l}\text { Cucumis callosus (Roettler) Cogn. } \\
\text { [BUBH0000095] }\end{array}$ & Cucurbitaceae & Gwmwri & Herb & $\mathrm{Wd}$ & $\mathrm{L}, \mathrm{F}$ & Raw & 2 & 0.047 \\
\hline 24. & Zingiber officinale Roscoe. [BUBH0000096] & Zingiberaceae & Haijeng & Herb & Do & $\mathrm{T}$ & Raw & 1 & 0.0238 \\
\hline 25. & Curcuma longa L. [BUBH0000002] & Zingiberaceae & Haldi & Herb & Do & $\mathrm{T}$ & Raw & 3 & 0.071 \\
\hline 26. & $\begin{array}{l}\text { Centipeda minima (L.) A. Br. \& Asch. } \\
\text { [BUBH0000097] }\end{array}$ & Asteraceae & Hatchew dongfang & Herb & $\mathrm{Wd}$ & WP & decoction & 1 & 0.0238 \\
\hline
\end{tabular}


Table 3. (Continued)

\begin{tabular}{|c|c|c|c|c|c|c|c|c|c|}
\hline Sl. No. & Scientific Name & Family & Local name & Habit & Habitat & Parts used & Formulation & FC & RFC \\
\hline 27. & Cissus quadrangularis L. [BUBH0000098] & Vitaceae & Hatjora & Scandent shrub & $\mathrm{Wd}$ & WP & Infusion & 1 & 0.0238 \\
\hline 28. & Ricinus communis L. [BUBH0000003] & Euphorbiaceae & Indi dongfang & Shrub & Do & $\mathrm{L}$ & Raw & 1 & 0.0238 \\
\hline 29. & $\begin{array}{l}\text { Drymaria cordata }(\mathrm{L} .) \text { Willd. ex Schult } \\
\text { [BUBH0000099] }\end{array}$ & Carryophyllaceae & Jabowsri & Herb & $\mathrm{Wd}$ & $\mathrm{L}$ & Raw & 1 & 0.0238 \\
\hline 30. & $\begin{array}{l}\text { Justicia gendarussa Burm. f. } \\
\text { [BUBH0000100] }\end{array}$ & Acanthaceae & Jaytrasi & Under shrub & $\mathrm{Wd}$ & $\mathrm{L}$ & Raw & 1 & 0.0238 \\
\hline 31. & Nigella sativa L. [BUBH0000101] & Ranunculaceae & Kaljeera & Herb & Do & S & Raw & 1 & 0.0238 \\
\hline 32. & $\begin{array}{l}\text { Andrographis paniculata (Burm.f.) Nees } \\
\text { [BUBH0000009] }\end{array}$ & Acanthaceae & Kalmech, Sirota & Herb & Do & $\mathrm{L}$ & Raw & 12 & 0.286 \\
\hline 33. & $\begin{array}{l}\text { Momordica charantica Linn. } \\
\text { [BUBH0000086] }\end{array}$ & Cucurbitaceae & Kerela gwkha, Udasi & Climber herb & Do & $\mathrm{L}$ & Raw & 1 & 0.0238 \\
\hline 34. & $\begin{array}{l}\text { Gymnopetalum chinense (Lour.) Merr. } \\
\text { [BUBH0000102] }\end{array}$ & Cucurbitaceae & Khaila & Herb & $\mathrm{Wd}$ & $\mathrm{L}, \mathrm{F}$ & Raw & 2 & 0.047 \\
\hline 35. & Meyna spinosa Roxb. ex ink [BUBH0000103] & Rubiaceae & $\begin{array}{l}\text { Khanthaokhra, Phanthao } \\
\text { goglang }\end{array}$ & Tree & Wd & $\mathrm{L}$ & Decoction & 2 & 0.047 \\
\hline 36. & Oroxylum indicum (L.) Kurz [BUBH0000012] & Bignoniaceae & Kharong & Tree & $\mathrm{Wd}$ & $\mathrm{R}, \mathrm{T}$ & Decoction & 1 & 0.0238 \\
\hline 37. & Rauvolfia tetraphylla L. [BUBH0000013] & Apocynaceae & Kharwkha & Shrub & Wd & $\mathrm{R}, \mathrm{B}, \mathrm{L}$ & Raw & 2 & 0.047 \\
\hline 38. & Amaranthus spinosus L. [BUBH0000104] & Amaranthaceae & Khuduna su, Khutra & Herb (spinous) & Wd & $\mathrm{L}$ & Decoction & 1 & 0.0238 \\
\hline 39. & Morinda citrifolia L. [BUBH00000016] & Rubiaceae & Khungkha gwkha & Tree & $\mathrm{Wd}$ & $\mathrm{L}$ & Decoction & 2 & 0.047 \\
\hline 40. & Solanum torvum Sw. [BUBH0000018] & Solanaceae & Khunthai nara & Shrub & $\mathrm{Wd}$ & $\mathrm{F}$ & Raw & 2 & 0.047 \\
\hline 41. & Averrhoa carambola L. [BUBH0000105] & Oxalidaceae & Khwrdwi, Kamrenga & Tree & Do & B & Decoction & 1 & 0.0238 \\
\hline 42. & Paederia foetida L. [BUBH0000015] & Rubiaceae & Kiphi bendwng & Climber & $\mathrm{Wd}$ & $\mathrm{L}$ & Raw & 1 & 0.0238 \\
\hline 43. & $\begin{array}{l}\text { Anthocephalus cadamba (Roxb.) Miq. } \\
\text { [BUBH0000106] }\end{array}$ & Rubiaceae & Kwdwm & Tree & Do & $\mathrm{F}$ & Raw & 1 & 0.0238 \\
\hline 44. & Citrus limon (L.) Osbeck [BUBH0000107] & Rutaceae & Lemon & Tree & Do & $\mathrm{S}$ & Decoction & 1 & 0.0238 \\
\hline 45. & Dimocarpus longan Lour. [BUBH0000108] & Sapindaceae & Lethekho, Hagrani lisu & Tree & Do & $\mathrm{R}$ & Decoction & 1 & 0.0238 \\
\hline 46. & $\begin{array}{l}\text { Syzygium aromaticum }(\text { L.) Merr. \& L.M.Perry } \\
\text { [BUBH0000079] }\end{array}$ & Myrtaceae & Long & Tree & Do & $\mathrm{S}$ & Decoction & 1 & 0.0238 \\
\hline 47. & $\begin{array}{l}\text { Hydrocotyle sibthorpioides Lam. } \\
\text { [BUBH0000019] }\end{array}$ & Araliaceae & Manimuni fisa & Herb & $\mathrm{Wd}$ & $\mathrm{L}, \mathrm{WP}$ & Raw & 8 & 0.1905 \\
\hline 48. & Centella asiatica L. [BUBH0000020] & Apiaceae & Manimuni gidir & Herb & $\mathrm{Wd}$ & $\mathrm{L}, \mathrm{WP}$ & Raw & 8 & 0.1905 \\
\hline 49. & Carica papaya L. [BUBH0000109] & Caricaceae & Mwiduful & Tree & Do & $\mathrm{R}$ & Decoction & 2 & 0.047 \\
\hline 50. & $\begin{array}{l}\text { Clerodendrum infortunatum } \mathrm{L} \text {. } \\
\text { [BUBH0000047] }\end{array}$ & Lamiaceae & Mwkhwna & Shrub & Wd & $\mathrm{L}$ & Decoction & 4 & 0.095 \\
\hline 51. & $\begin{array}{l}\text { Lindernia crustacea (L.) F. Muell. } \\
\text { [BUBH0000048] }\end{array}$ & Linderniaceae & Na bikhi & Herb & Wd & $\mathrm{L}$ & Decoction & 1 & 0.0238 \\
\hline 52. & Citrus grandis (L.) Osbeck. [BUBH0000064] & Rutaceae & Nareng jumbra & Tree & Do & $\mathrm{F}$ & Maceration & 2 & 0.047 \\
\hline 53. & Azadirachta indica A. Juss. [BUBH0000051] & Meliaceae & Neem & Tree & Do & $\mathrm{L}$ & $\begin{array}{l}\text { Raw, } \\
\text { decoction }\end{array}$ & 3 & 0.071 \\
\hline 54. & Asparagus racemosus Willd. [BUBH0000063] & Asparagaceae & Nilikhor & Climber & $\mathrm{Wd}$ & $\mathrm{R}$ & Raw & 2 & 0.047 \\
\hline 55. & Cajanus cajan (L.) Millsp. [BUBH0000110] & Leguminosae & Ohor, Khokling & Shrub & Do & $\mathrm{L}, \mathrm{S}$ & Raw & 3 & 0.071 \\
\hline 56. & $\begin{array}{l}\text { Bryophyllum pinnatum }(\mathrm{Lam} .) \text { Oken } \\
\text { [BUBH0000057] }\end{array}$ & Crassulaceae & Paat gaja & Herb & Do & $\mathrm{L}$ & Decoction & 1 & 0.0238 \\
\hline 57. & Ficus riligiosa Linn. [BUBH0000082] & Moraceae & Phakhri dongfang & Tree & Wd & B & Raw & 1 & 0.0238 \\
\hline 58. & Ocimum basilicum L. [BUBH0000111] & Lamiaceae & Ramtulusi & Herb & Do & $\mathrm{L}$ & Raw & 1 & 0.0238 \\
\hline 59. & Allium sativum L. [BUBH0000112] & Amaryllidaceae & Sambram gufur & Herb & Do & $\mathrm{T}$ & Raw & 1 & 0.0238 \\
\hline 60. & Achyranthes aspera L. [BUBH0000046] & Amaranthaceae & Samper ultha, Ultasur & Herb & Wd & $\mathrm{L}$ & Decoction & 2 & 0.047 \\
\hline 61. & Terminalia chebula Retz. [BUBH0000062] & Combretaceae & Silikha & Tree & Do & $\mathrm{F}$ & Raw & 5 & 0.119 \\
\hline 62. & Piper longum L. [BUBH0000085] & Piperaceae & Simfri fithai & Herb & Do & $\mathrm{F}$ & Raw & 1 & 0.0238 \\
\hline 63. & Bixa orellana L. [BUBH0000113] & Bixaceae & Sindoor dongfang & Tree & Wd & B & Infusion & 1 & 0.0238 \\
\hline 64. & Oxalis corniculata L. [BUBH0000114] & Oxalidaceae & Singri mwkhi fisa & Herb & $\mathrm{Wd}$ & $\mathrm{L}$ & Decoction & 3 & 0.071 \\
\hline 65. & $\begin{array}{l}\text { Alstonia scholaris (L.) R. Br. } \\
\text { [BUBH0000040] }\end{array}$ & Apocynaceae & Sithona & Tree & $\mathrm{Wd}$ & B & Infusion & 4 & 0.095 \\
\hline 66. & Psidium guajava L. [BUBH0000041] & Myrtaceae & Sofari & Tree & Do & $\mathrm{L}$ & Raw & 8 & 0.1905 \\
\hline
\end{tabular}


Table 3. (Continued)

\begin{tabular}{|c|c|c|c|c|c|c|c|c|c|}
\hline SI. No. & Scientific Name & Family & Local name & Habit & Habitat & Parts used & Formulation & FC & RFC \\
\hline 67. & Kaempferia galangal L. [BUBH0000115] & Zingiberaceae & Sompera & Herb & Do & $\mathrm{T}$ & Raw & 1 & 0.0238 \\
\hline 68. & Cassia fistula L. [BUBH0000043] & Leguminosae & Sonalu & Tree & $\mathrm{Wd}$ & $\mathrm{L}$ & Raw & 1 & 0.0238 \\
\hline 69. & Streblus asper Lour. [BUBH0000116] & Moraceae & Soura & Tree & $\mathrm{Wd}$ & St & Raw & 1 & 0.0238 \\
\hline 70. & Mangifera indica L. [BUBH0000117] & Anacardiaceae & Thaijow & Tree & Do & S & Raw & 1 & 0.0238 \\
\hline 71. & Spondias pinnata (L.f.) Kurz [BUBH0000118] & Anacardiaceae & Thaisuri & Tree & Do & B & Raw & 1 & 0.0238 \\
\hline 72. & Musa balbisiana Colla. [BUBH2018067] & Musaceae & Athia thalir & Tree & Do & $\mathrm{C}$ & Raw & 3 & 0.071 \\
\hline 73. & Ocimum sanctum L. [BUBH2018045] & Lamiaceae & Tulungsi & Undershrub & Do & $\mathrm{L}$ & Raw & 1 & 0.0238 \\
\hline 74. & Ocimum gratissimum L. [BUBH0000119] & Lamiaceae & Tulungsi gidir & Shrub & Do & $\mathrm{L}$ & Raw & 1 & 0.0238 \\
\hline 75. & $\begin{array}{l}\text { Acmella paniculata (Wall. ex DC.) R.K.Jansen } \\
\text { [BUBH2018007] }\end{array}$ & Asteraceae & Usumwi, jhari & Herb & $\mathrm{Wd}$ & $\mathrm{L}$ & Raw & 1 & 0.0238 \\
\hline
\end{tabular}

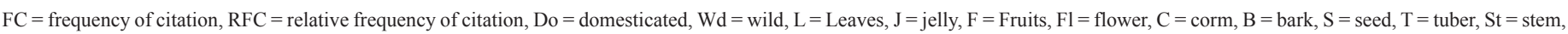
$\mathrm{R}=$ roots, $\mathrm{WP}=$ whole plant, $\mathrm{En}=$ endosperm.

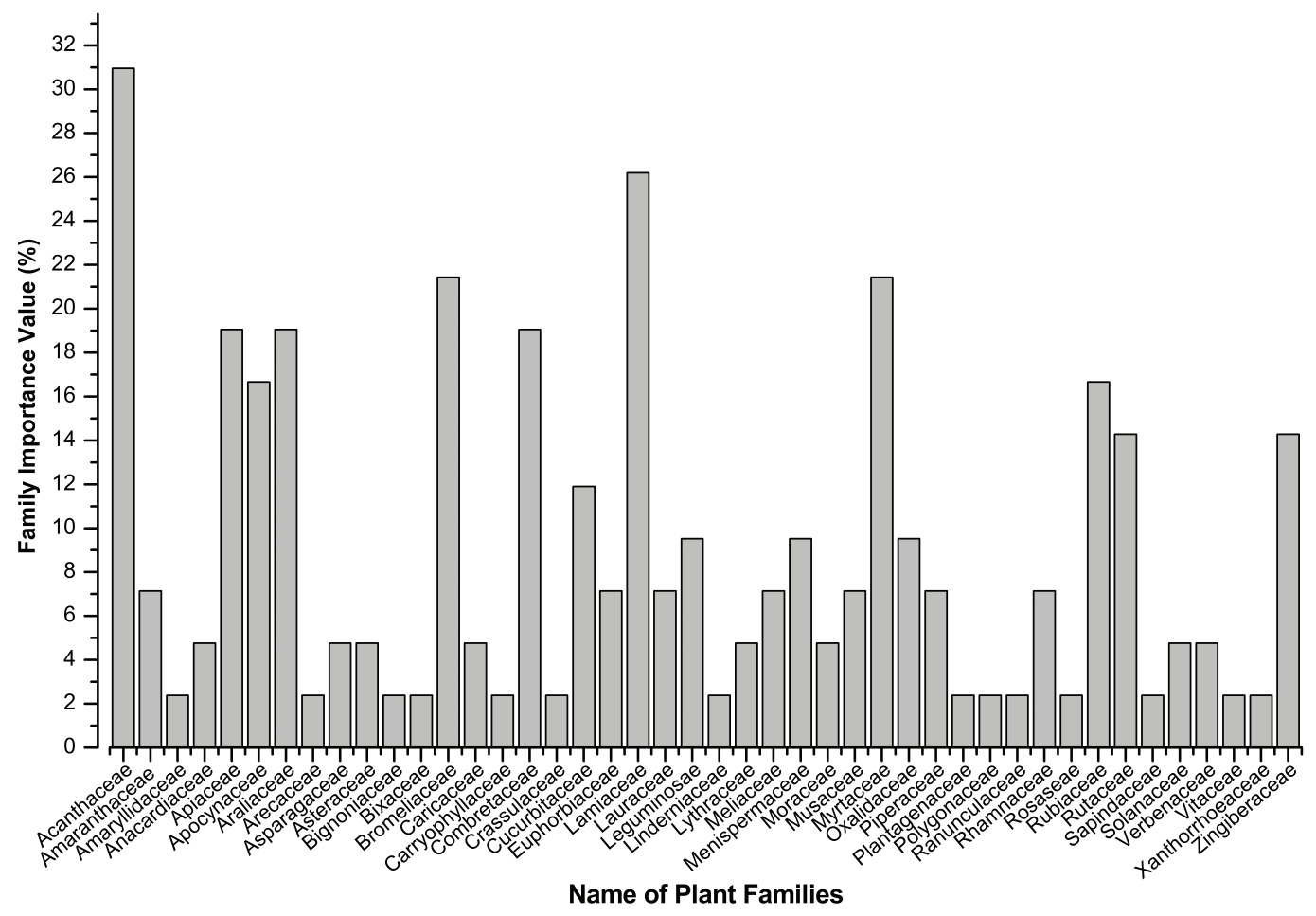

Figure 2. Graph showing the popularity of different Families of plants in Udalguri district of Assam having anthelmintic property.

However, $27 \%$ (43 citations) of the plants do not have any repetitions among the informants. A total of 75 plants species, belonging to 67 genera and 44 families were recorded from Udalguri district that are consumed as deworming medicines. The plants belonging to 16 families are found to be more common and have more than one citation. Andrographis paniculata was found to be the most popular plant species $(\mathrm{FC}=12, \mathrm{RFC}=0.286)$ followed by Ananas comosus $(\mathrm{FC}=9, \mathrm{RFC}=0.214)$, Hydrocotyle sibthorpioides $(\mathrm{FC}$ $=8, \mathrm{RFC}=0.190)$, Centella asiatica $(\mathrm{FC}=8, \mathrm{RFC}=0.190)$, and Psidium guajava $(\mathrm{FC}=8, \mathrm{RFC}=0.190)$. Similarly, out of 45 plant families, Acanthaceae was seen to be the most popular family with FIV value 32\% followed by Bromeliaceae, Myrtaceae, Apiaceae, Araliaceae, and Lamiaceae with more than 15\% FIV value (Fig. 2). Thirteen plant families do not seem to have any popularity among the traditional healers in terms of FIV value. However, in terms of plants species used under a single family, Rubiaceae was seen to be important with eight number of plant species, followed by Lamiaceae (six species), Zingiberaceae (four speciess), Cucurbitaceae and Apocynaceae (three species each), and Verbenaceae, Piperaceae, Oxalidaceae, Myrtaceae, Leguminosae, Lauraceae, Euphorbiaceae, Acanthaceae, Amaranthaceae, Anacardiaceae, and Asteraceae (two species each). However, from 29 families only one plant citation was reported. It has been found that most of the plants $(53 \%)$ cited are wild in habitat, while others are domesticated. Our study also revealed that majority of the plants belongs to trees (39\%). In our study, we also found that $37 \%$ of the plant species are herbs, $20 \%$ are shrubs, and $4 \%$ are climbers. Figure 3 shows the citation of the plants by different informants. 


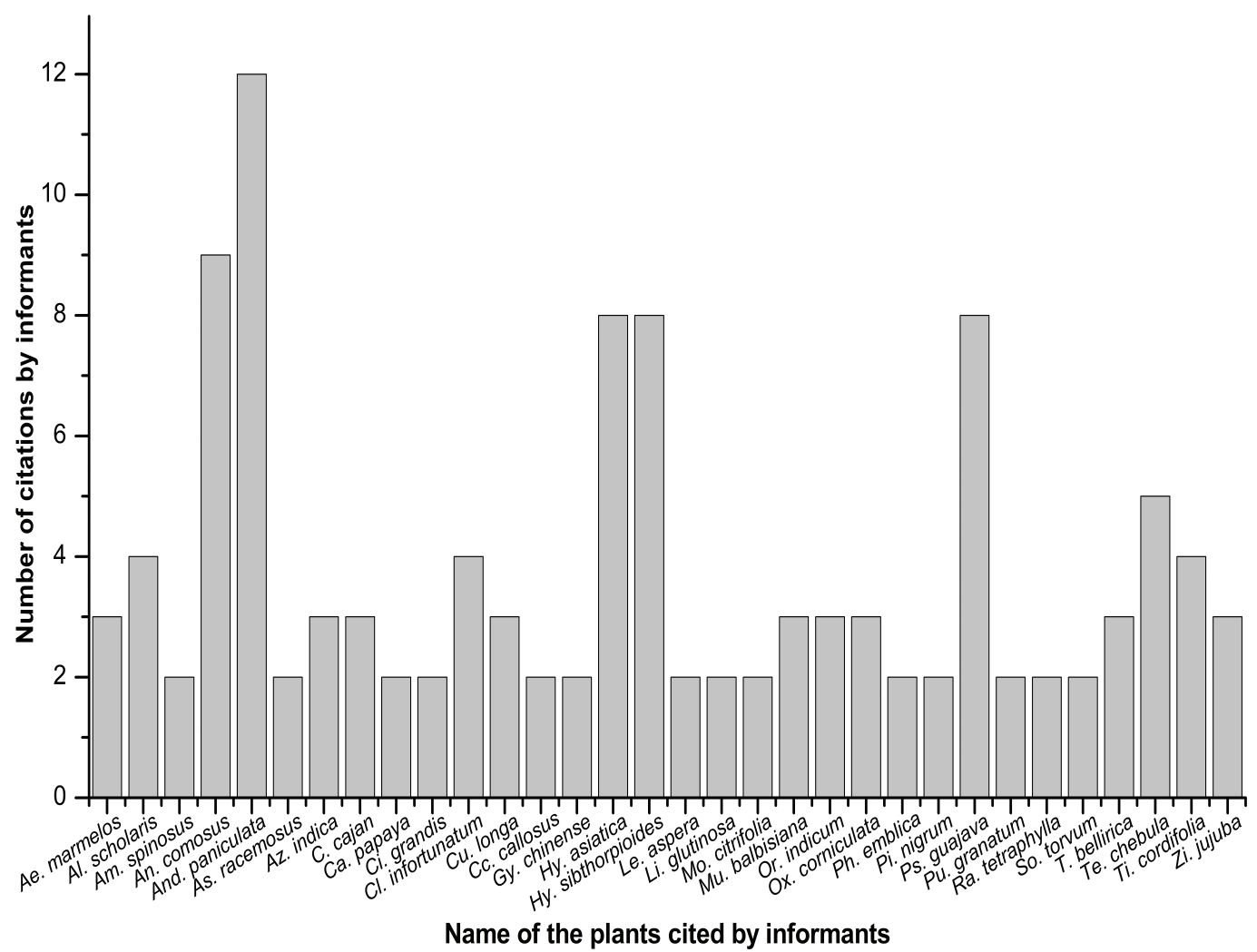

Figure 3. List of plants citations by different informants of Udalguri district of Assam.

Our survey reported a total of 75 species of plants used in TM system as deworming medicines. Ananas comosus, A. paniculata, $C$. asiatica, $H$. sibthorpioides, and Psidium guajava were found to be the most popular plants used as an anthelmintic agent among the tribal communities of Udalguri district. The traditional popularity has also been confirmed experimentally by many investigators (Molla and Bandyopadhyay, 2014; PinaVázquez et al., 2017). Similarly, plants having two or more citations, such as Aegle marmelos (Singh et al., 2012), Alstonia scholaris (Panda et al., 2017), and Clerodendrum infortunatum (Swargiary et al., 2016) also possess experimental evidence about anthelmintic activity. Out of 75 plant species reported by informants, $65 \%$ are having scientific and experimental evidence of anthelmintic property. The presence of such experimental evidence about the medicinal plants traditionally practiced by traditional healers suggests the efficiency and popularity of ethnomedicine. In a similar kind of research, several species of plants were reported from Cachar district of Assam having anthelmintic property (Das et al., 2008). Similarly, Sharma and Sharma (2010) reported many anthelmintic plants from Sonapur area of Kamrup district of Assam. Many plants from northeast India were investigated by Lyndem et al. (2008) showing effective anthelmintic agents. It can therefore be believed that although traditional healers do not perform any laboratory experiment, they have some kind of customary procedure by which they formulate their herbal doses.

Different plant parts are generally used in the preparation of herbal remedies. Among the plant parts used, leaves were found to be the most commonly used for the preparation of deworming
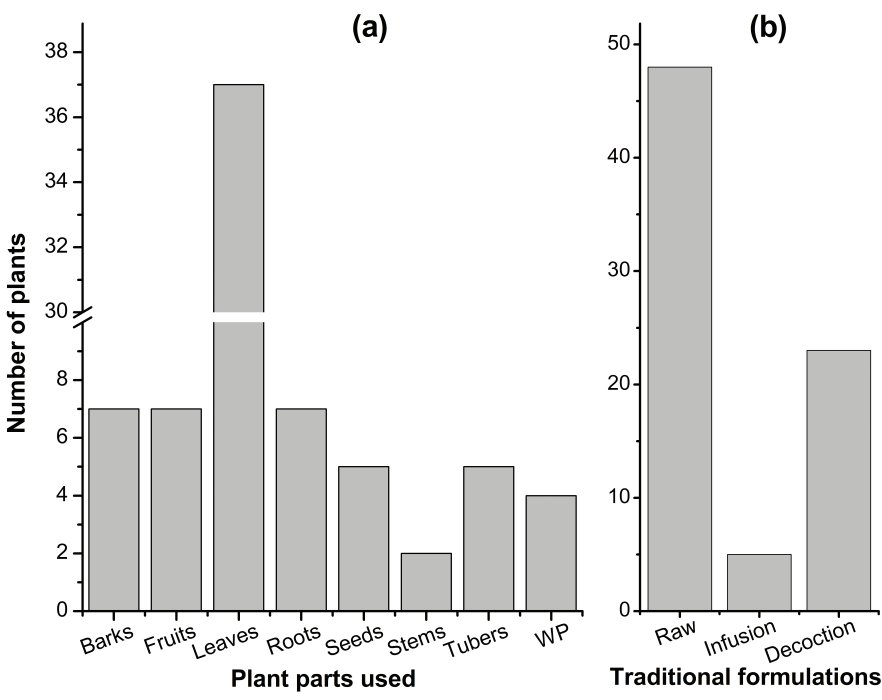

Figure 4. (a) Plant parts used in the traditional herbal formulation and (b) types of traditional formulations practiced. WP- whole plant.

herbal medicines (Fig. 4a). Unlike others, for three plants, namely, Musa balbisiana, Cocos nucifera, and Clerodendrum indicum, the corm, endosperm, and flower were found to be used for herbal formulations. Our survey also found that Rauvolfia tetraphylla is the most common plant in terms of parts used where all the leaves, barks, and roots were found to be used. Our survey also found that four plants, viz., Cen. minima, Cissus quadrangularis, 
H. sibthorpioides, and C. asiatica, the whole plant parts was used for the herbal formulations. Regarding the herbal formulations, it has been seen that tribal communities of Udalguri district follows three methods of herbal formulations-decoction, infusion, and raw. Most of the time, traditional healers use raw plants for the preparation of herbal medicines (Fig. 4b). Trees are the most commonly used plants used by the herbal healers to cure helminthiasis followed by herbs, shrubs, and climbers. Because of its easy accessibility and availability, leaves tend to be the most commonly used plant parts. Many such studies have reported similar use of traditionally used medicinal plants (Choudhury et al., 2015; Raj et al., 2018). Although there are variations in the nature of herbal formulations among the traditional healers, three main traditional formulations are practiced by tribal communities of Udalguri district - raw, infusion, and decoction. Similar to our findings, decoction, extract, infusion, powder, and juice were found to be the main traditional formulations practiced by Neelum Valley, Pakistan (Ahmad et al., 2017).

\section{CONCLUSION}

Our study revealed that the ethnomedicinal knowledge for the treatment of helminth infection is prominent among the tribal communities of Udalguri district of Assam. A variety of medicinal plants are found to be used in traditional medicine system to treat helminthiasis. This study provides the documentation of the medicinal plants used for traditional healthcare. The literature survey has shown that most of the medicinal plants reported in the present study have scientific validation about their anthelmintic activity. Our survey also revealed that there is no precise method for formulating herbal remedies, but doses are altered according to their age and severity of the illness. The documented medicinal plants can serve as a database for future work or scientific validation. Furthermore, this study opens the door to scientific approach, which could lead to the discovery of new drugs, with lesser side effects.

\section{ACKNOWLEDGMENT}

AS would like to thank DST-SERB, Govt. of India for providing financial assistance (EEQ/2017/000071). The authors would also like to thank the traditional healer and elderly people for providing ethnomedicinal information. The authors also acknowledge to Dr. Sanjib Baruah Asstt. Prof., Department of Botany and Mr. Sanswrang Basumatary, Research Scholar, Department of Botany for helping scientific validation of the sample plants. The authors also acknowledge the infrastructural facilities provided by Department of Zoology, Bodoland University.

\section{CONFLICT OF INTEREST}

The authors declare that they have no conflict of interest.

\section{REFERENCES}

Ahmad KS, Hamid AH, Nawaz F, Hameed M, Ahmad F, Deng J, Akhtar N, Wazarat A, Mahroof S. Ethnopharmacological studies of indigenous plants in Kel village, Neelum Valley, Azad Kashmir, Pakistan. J Ethnobiol Ethnomed, 2017; 13:68.

Ahmad L, Semotiuk A, Zafar M, Ahmad M, Sultana S, Liu QR, Zada MP, Ul Abidin SZ, Yaseen G. Ethnopharmacological documentation of medicinal plants used for hypertension among the local communities of DIR Lower, Pakistan. J Ethnopharmacol, 2015; 175:138-46.
Akhtar MS, Iqbal Z, Khan MN, Lateef M. Anthelmintic activity of medicinal plants with particular reference to their use in animals in the Indo-Pakistan subcontinent. Small Rumin Res, 2000; 38:99-107.

Choudhury RC, Dutta Choudhury M, Ningthoujam SS, Das D, Nath D, Das Talukdar A. Ethnomedicinal plants used by traditional healers of North Tripura district, Tripura, North East India. J Ethnopharmacol, $2015 ; 166: 135-48$

Das AK, Dutta BK, Sharma GD. Medicinal plants used by different tribes of Cachar district, Assam. Indian J Tradit Know, 2008; 7:446-54.

Fayaz M, Jain AK, Bhat MH, Kumar A. Ethnobotanical survey of Daksum forest range of Anantnag District, Jammu and Kashmir, India. J Herbs Spices Med Plants, 2019; 25(1):55-67.

Hussain W, Ullah M, Dastagir G, Badshah L. Quantitative ethnobotanical appraisal of medicinal plants used by inhabitants of lower Kurram, Kurram agency, Pakistan. Avicenna J Phytomed, 2018; 8:313-29.

Lyndem LM, Tandon V, Das B. Anthelmintic efficacy of medicinal plants from Northeast India against hookworms: an in vitro study on Ancylostoma ceylanicum. Pharmacologyonline, 2008; 3:697-707.

Maphosa V, Masika PJ. Ethnoveterinary uses of medicinal plants: a survey of plants use in the ethnoveterinary control of gastrointestinal parasites of goats in the Eastern Cape Province, South Africa. Pharm Biol, 2010; 48:697-702.

Molla SH, Bandyopadhyay PK. In-vitro anthelmintic activity of Psidium guajava against sheep gastrointestinal nematode, Haemonchus contortus. Environ Ecol, 2014; 32:616-21.

Muthee JK, Gakuya DW, Mbaria JM, Kareru PG, Mulei CM, Njonge FK. Ethnobotanical study of anthelmintic and other medicinal plants traditionally used in Loitoktok district of Kenya. J Ethnopharmacol, $2015 ; 135: 15-21$.

Panda SK, Padhi L, Leyssen P, Liu M, Neyts J, Luyten W. Antimicrobial, anthelmintic, and antiviral activity of plants traditionally used for treating infectious disease in the Similipal Biosphere Reserve, Odisha, India. Front Pharmacol, 2017; 8:658; doi:10.3389/fphar.2017.00658

Pina-Vázquez DM, Mayoral-Pena Z, Gómez-Sanchez $M$, Salazar-Olivo LA, Arellano-Carbajal F. Anthelmintic effect of Psidium guajava and Tagetes erecta on wild-type and levamisole-resistant Caenorhabditis elegans strains. J Ethnopharmacol, 2017; 202:92-6.

Raj AJ, Biswakarma S, Pala NA, Shukla G, Vineeta, Kumar M, Chakravarty S, Bussmann RW. Indigenous uses of ethnomedicinal plants among forest-dependent communities of Northern Bengal, India. J Ethnobiol Ethnomed, 2018; 14:8.

Ritter RA, Monteiro MVB, Monteiro FOB, Rodrigues ST, Soares ML, Silva JC, Palha Md, Biondi GF, Rahal SC, Tourinho MM. Ethnoveterinary knowledge and practices at Colares island, Para State, Eastern Amazon, Brazil. J Ethnopharmacol, 2012; 114:346-52.

Sharma R, Sharma HK. Ethnomedicines of Sonapur, Kamrup district. Indian J Tradit Know, 2010; 9:163-5.

Shikov A, Pozharitskaya O, Makarov V, Wagner H, Verpoorte R, Heinrich M. Medicinal plants of the Russian pharmacopoeia; their history and applications. J Ethnopharmacol, 2014; 154:481-536.

Singh A, Nautiyal MC, Kunwar RM, Bussmann RW. Ethnomedicinal plants used by local inhabitants of Jakholi block, Rudraprayag district, Western Himalaya, India. J Ethnobiol Ethnomed, 2017; 13:49.

Singh K, Agrawal KK, Gupta JK. Comparative anthelmintic activity of Aegle marmelos Linn leaves and pulp. J Pharm, 2012; 2(3): $395-7$.

Swargiary A, Daimari A, Daimari M, Basumatary N, Narzary E. Phytochemicals, antioxidant, and anthelmintic activity of selected traditional wild edible plants of lower Assam. Indian J Pharmacol, 2016; 48:418-23.

Tardio J, Pardo-de Santayana M. Cultural importance indices: a comparative analysis based on the useful wild plants of southern Cantabria (northern Spain). J Eco Bot, 2008; 62:24-39. 
Teklehaymanot T. An ethnobotanical survey of medicinal and edible plants of Yalo Woreda in Afar regional state, Ethiopia. J Ethnobiol Ethnomed, 2017; 13:40.

Vitalini, S, Iriti, M, Puricelli, C, Ciuchi, D, SegaleA, Fico G. Traditional knowledge on medicinal and food plants used in Val San Giacomo (Sondrio, Italy) an alpine ethnobotanical study. J Ethnopharmacol, 2013; 145:517-29.

Wangchuk P, Giacomin PR, Pearson MS, Smout MJ, Loukas A. Identification of lead chemotherapeutic agents from medicinal plants against blood flukes and whipworms. Sci Rep, 2016; 6:32101.
How to cite this article:

Swargiary A, Daimari M, Roy MK. Survey and documentation of anthelmintic plants used in traditional medicine system of tribal communities of Udalguri district of Assam, India. J Appl Pharm Sci, 2020; 10(1):046-054. 